\title{
The effect of magnetic stimulation on the osteogenic and chondrogenic differentiation of human stem cells derived from the adipose tissue (hASCs)
}

\author{
João Lima ${ }^{\mathrm{a}, \mathrm{b}}$, Ana I. Gonçalves ${ }^{\mathrm{a}, \mathrm{b}}$, Márcia T. Rodrigues ${ }^{\mathrm{a}, \mathrm{b}}$, Rui L. Reis ${ }^{\mathrm{a}, \mathrm{b}}$, \\ Manuela E. Gomes ${ }^{\mathrm{a}, \mathrm{b}, *}$ \\ a 3Bs Research Group-Biomaterials, Biodegradables and Biomimetics, University of Minho, Guimarães, Portugal \\ ${ }^{\mathrm{b}}$ ICVS/3Bs-PT Government Associate Laboratory, Braga/Guimarães, Portugal
}

\section{A R T I C L E I N F O}

\section{Article history:}

Received 2 March 2015

Received in revised form

27 May 2015

Accepted 30 May 2015

Available online 6 June 2015

Keywords:

Adipose-derived stem cells

Osteogenic differentiation

Chondrogenic differentiation

Magnetic nanoparticles

Tissue engineering

Regenerative medicine

\begin{abstract}
A B S T R A C T
The use of magnetic nanoparticles (MNPs) towards the musculoskeletal tissues has been the focus of many studies, regarding MNPs ability to promote and direct cellular stimulation and orient tissue responses. This is thought to be mainly achieved by mechano-responsive pathways, which can induce changes in cell behavior, including the processes of proliferation and differentiation, in response to external mechanical stimuli. Thus, the application of MNP-based strategies in tissue engineering may hold potential to propose novel solutions for cell therapy on bone and cartilage strategies to accomplish tissue regeneration.

The present work aims at studying the influence of MNPs on the osteogenic and chondrogenic differentiation of human adipose derived stem cells (hASCs). MNPs were incorporated in hASCs and cultured in medium supplemented for osteogenic and chondrogenic differentiation. Cultures were maintained up to 28 days with/without an external magnetic stimulus provided by a magnetic bioreactor, to determine if the MNPs alone could affect the osteogenic or chondrogenic phenotype of the hASCs.

Results indicate that the incorporation of MNPs does not negatively affect the viability nor the proliferation of hASCs. Furthermore, Alizarin Red staining evidences an enhancement in extracellular (ECM) mineralization under the influence of an external magnetic field. Although not as evident as for osteogenic differentiation, Toluidine blue and Safranin-O stainings also suggest the presence of a cartilage-like ECM with glycosaminoglycans and proteoglycans under the magnetic stimulus provided.

Thus, MNPs incorporated in hASCs under the influence of an external magnetic field have the potential to induce differentiation towards the osteogenic and chondrogenic lineages.
\end{abstract}

(c) 2015 Elsevier B.V. All rights reserved.

\section{Introduction}

Bone and cartilage defects are a clinical problem that affects a multitude of people worldwide. Bone tissue has innate regeneration capacity granting this tissue the capacity to self-repair minor injuries. However, large bone defects are not fully healed without treatments, such as surgical procedures, whose outcomes have limited success in a long-term basis. Cartilage, on the contrary, exhibits a naturally limited regeneration capacity associated to the lack of vascularization hindering its healing process. Current strategies in the treatment of bone and cartilage, namely auto- and

\footnotetext{
* Correspondence to: at: 3Bs Research Group-Biomaterials, Biodegradables and Biomimetics, University of Minho, Headquarters of the European Institute of Excellence on Tissue Engineering and Regenerative Medicine, Guimarães 4806-909, Portugal.

E-mail address: megomes@dep.uminho.pt (M.E. Gomes).
}

allo-grafting, and implantation of metallic prosthesis [1,2] are unable to fully restore complete tissue function, and evidence severe disadvantages that include limitation of autologous donor sites, risks of disease transmission and immunosuppression, risks of infection and extrusion of the prosthesis and lack of functionality [3].

Tissue engineering (TE) offers novel approaches to treat and regenerate bone and cartilage defects. In the past few years, magnetic nanoparticles (MNPs) have gained a prominent position in the biomedical field, and increasingly used as contrast agents for magnetic resonance imaging or drug delivery systems [4]. In the TE field, MNPs are being studied as magneto-mechanical stimulators/activators of cell arrays, in mechano-sensitive ion channels and magnetic cell sorting procedures, and in mechanisms of controlled cell proliferation and differentiation [5]. Moreover, the application of MNPs in combination with TE strategies could result in the development of innovative solutions to stimulate cells at a 
nanoscale, that is, at the cellular level, influencing cellular processes and responses such as cell migration or differentiation. Mesenchymal stem cells are often considered a promising cell source due to their intrinsic and well described properties in many studies aiming at cell-based therapies. Among them, human adipose-derived stem cells (hASCs) can be harvested with minimal invasive procedures and isolated and expanded to high numbers. Moreover, hASCs have long-term genetic stability and have demonstrated potential to undergo adipogenic, neurogenic, osteogenic and chondrogenic differentiation [6-10].

Considering the impact of MNPs in cell guidance and behavior aiming at cell based strategies, this study proposes to investigate the influence of MNPs in promoting hASCs differentiation towards osteogenic and chondrogenic lineages, under the actuation of a remote mechano-magnetic stimulus provided by an external magnetic field.

For that purpose, MNPs were incorporated in hASCs, and cultured in basic medium or media containing osteogenic or chondrogenic specific supplements. The influence of the MNPs per se or under the actuation of an external magnetic stimulus generated by a bioreactor apparatus in synergy with medium biochemical factors was also assessed in the differentiation process of hASCs.

Cell viability and proliferation levels were weekly evaluated by MTS and DNA quantification assays, respectively for up to 28 days. Bone and cartilage lineage commitment was assessed through specific histological stains to detect markers associated to native bone or cartilage extracellular matrices.

\section{Materials and methods}

\subsection{Materials}

Magnetic nanoparticles (MNPs) selected for this study were commercially available at Micromod (Germany) under a product name nanomag ${ }^{\mathbb{R}}-C L D-r e d F$ (23-00-102). These MNPs with approximately $100 \mathrm{~nm}$ in diameter are cross-linked dextran iron oxide composite particles with an iron oxide core [CAS: 1317-61-9] of about $75-80 \mathrm{wt} \%$, and with a polydispersity index $<0.2$. These particles also include optical properties as being red fluorescent. Based on previous studies performed (data not shown) MNPs were re-suspended in basic culture medium at $370 \mu \mathrm{g} / \mathrm{ml}$. MNPs were handled in controlled conditions and operated under aseptic conditions.

\subsection{Assessment of MNPs stability in the culture medium}

In order to test the stability of these particles in suspension in the culture medium, a turbidity assay was monitored over a period up to $24 \mathrm{~h}$, namely $0,1.5 \mathrm{~h}, 16 \mathrm{~h}$ and $24 \mathrm{~h}$, with a MNP concentration of $370 \mu \mathrm{g} / \mathrm{ml}$. MNPs suspended in culture medium or in PBS, as in the original suspension provided by Micromod, was analyzed in quadruplicates.

The turbidity of MNPs suspension was measured at $450 \mathrm{~nm}$ $[11,12]$ using a microplate reader (Bio-tek, synergie HT).

\subsection{Human adipose derived stem cells isolation and expansion}

Human adipose derived stem cells (hASCs) were obtained from lipoaspirate samples following a protocol previously established with the Department of Plastic Surgery of Hospital da Prelada, Porto, Portugal. Samples were collected following informed consent and the protocol of ethics. Cells were isolated as described elsewhere [13]. Briefly, samples were digested using $0.2 \%$ collagenase type II (Sigma) in phosphate buffered saline (PBS), for $45 \mathrm{~min}$, at $37^{\circ} \mathrm{C}$ under stirring. A lysis buffer was used to remove the erythrocytes present. Adherent cells were then cultured and expanded in a basic medium composed of $\alpha$-minimal essential medium ( $\alpha$-MEM; Gibco) with 10\% Fetal Bovine Serum (FBS) (Gibco; heat-inactivated), 1\% Antibiotic-Antimycotic solution (A/B, Invitrogen) and Sodium Bicarbonate (Sigma). Medium was changed three times per week. Then, cells were cryopreserved in a solution containing 90\% FBS and 10\% dimethyl sulphoxide (DMSO CryoSure, Wak-Chemie Medical GMBH) until further use. Cells were used in passage 2 .

\subsection{Determination of MNPS incorporation in hASCS}

\subsubsection{Microscopy analysis}

In a previous study, we observed that hASCs were able to incorporate the particles even in the absence of a magnetic force stimulus provided by the bioreactor (Magnefect Nano II, Nanotherics) (unpublished data). Thus, to verify these outcomes for the differentiation study, hASCs were cultured in adherent 24-well plates at a density of 10,000 cells/well, and MNPs ressuspended in basal medium. After an overnight incubation, hASCs were washed in PBS and stained with Phalloidin-Tetramethylrhodamine B isothiocyanate (Phalloidin) solution, which was prepared accordingly to manufacturer's instructions (P1951, Sigma; dilution 1:200), followed by buffered formalin solution (43.05-k01009, INOPAT) fixation for $30 \mathrm{~min}$, and by 4,6-Diamidino-2-phenyindole, dilactate (DAPI, $5 \mu \mathrm{g} / \mu \mathrm{l}$, D9564, Sigma) stain for $10 \mathrm{~min}$.

Moreover, cells were stained with a solution of $20 \%$ hydrochloric acid (VWR) and 10\% potassium ferrocyanide (P3289, Sig$\mathrm{ma}$ ) to obtain a bright blue pigment, called prussian blue or ferric ferrocyanide, used for iron detection. Briefly, cells incorporating MNPs were immersed in a solution of equal parts of hydrochloric acid and potassium ferrocyanide for $20 \mathrm{~min}$, followed by washing in distilled water and counterstained with nuclear fast red (Sigma) for $7 \mathrm{~min}$. Samples were then rinsed twice in distilled water and kept in PBS until visualization. hASCs were then analyzed under a transmitted and reflected light microscope (Zeiss, Imager Z1M) for the detection and co-location of MNPs and the cells.

The MNPs within the cells were also screened by scanning electron microscopy (SEM). After MNPs incubation, hASCs were rinsed in PBS, fixed in formalin and dehydrated in a series of ethanol concentrations (30\%, 50\%, 70\%, 80\%, 90\% and 100\% ethanol) under mild agitation. Afterwards, the specimens were let to air-dry in a hood over night before sputter coated with gold. SEM observation was performed with a JSM-6010LV equipment (JEOL, Japan) equipped with an energy dispersive spectroscope (INCAXAct, PentaFET Precision, Oxford Instruments, UK).

\subsubsection{ICP spectrometry}

The efficiency of MNPs incorporation in hASCs was further verified by measuring the levels of iron released into the culture medium after ASCs incubation with the MNPs. Briefly, the iron released was evaluated by an ICP spectrometer (JY2000-2, Jobin Yvon, Horiba, Japan) in culture medium samples collected after $24 \mathrm{~h}$ of incubation of the cells with MNPs, and filtered $(0.22 \mu \mathrm{m}$, VWR) prior to analysis. Due to the presence of iron in $\alpha$-MEM composition, a sample of $\alpha$-MEM medium was used as blank solution. An iron (Fe, $1000 \mu \mathrm{g} / \mathrm{mL}$ ) standard solution (13830, Specpure $\left.{ }^{\circledR}\right)$ was used to prepare the standard concentration solutions.

\subsection{Assessment of cell viability (MTS assay) and cell content (double strand DNA quantification assay)}

After each of the selected time-point (7, 14, 21 and/or 28 days), culture medium was removed, samples rinsed with PBS and their metabolic activity assessed using a MTS assay performed following manufacturer instructions. Concisely a solution of MTS reagent (3- 
(4,5-dimethylthiazol-2-yl)-5-(3-carboxymethoxyphenyl)-2-(4-sulfophenyl)-2H-tetrazolium, inner salt, Promega Corporation) and DMEM basic medium without phenol red and FBS were prepared in a 1:5 ratio followed by a 3 -h incubation at $37^{\circ} \mathrm{C}$ in a humidified atmosphere containing $5 \% \mathrm{CO}_{2}$. This assay is a colorimetric method for determining the number of viable cells and is based on the bioreduction of the substrate into a brown formazan product accomplished by dehydrogenase enzymes in metabolically active cells. Afterwards, the supernatant was transferred to 96-well plates and the optical density (OD) determined at $490 \mathrm{~nm}$ in a microplate reader (Bio-tek, synergie HT).

Cell proliferation was determined by a fluorimetric doublestrand DNA quantification kit (Quant-iT PicoGreen dsDNA reagent, Molecular Probes, Invitrogen) after 7, 14, 21 and/or 28 days in culture.

After a PBS rinsing step, the cells in monolayer (osteogenic cultures) were detached and pellets (chondrogenic cultures) were collected into microtubes containing $1 \mathrm{~mL}$ of ultrapure water. All samples were stored at $-80^{\circ} \mathrm{C}$, until usage.

For dsDNA analysis, samples were thawed and sonicated for 15 min. Samples were placed on a white opaque 96-well plate (Labclinics), prior to the addition of a PicoGreen solution (1:200 in TE buffer). The plate was incubated for $10 \mathrm{~min}$ in the dark, and the fluorescence was measured on a microplate reader with an excitation of $485 / 20 \mathrm{~nm}$ and an emission of $528 / 20 \mathrm{~nm}$. A standard curve was made with standards ( 0 to $2 \mu \mathrm{g} / \mathrm{mL}$ ) to determine dsDNA concentration. Samples and standards were made in triplicate.

\subsection{MNPs and external magnetic field stimulation in the differ- entiation process of hASCS}

\subsubsection{Osteogenic differentiation of hASCS}

hASCs were cultured in standard 2D monolayer cultures. Twothousand (2000) cells were seeded in each well of a 24 well culture plate (Falcon). MNPs were added to cells $24 \mathrm{~h}$ after the seeding so that cells were able to attach before the incubation with MNPs. Basic medium was replaced by osteogenic supplemented culture medium 4 days after the incorporation of MNPs. Osteogenic medium was composed of $\alpha$-MEM, $10 \%$ FBS, $1 \%$ A/B, $\beta$-Glicerophosphate (10 mM, Sigma), Ascorbic Acid (50 $\mu \mathrm{g} / \mathrm{mL}$, Sigma) and Dexamethasone $\left(10^{-9} \mathrm{M}\right.$, Sigma).

hASCs cultured in basic medium were considered a negative control of osteogenic differentiation.

hASCs were kept in culture for up to 21 days, and the osteogenic medium was changed twice a week. Samples for MTS, DNA and histological assays were weekly collected up to 21 days in culture.

\subsubsection{Chondrogenic differentiation of hASCs}

Human ASCs were assembled in 3D pellets, a standard procedure to promote in vitro chondrogenic differentiation. Pellets were formed with 250,000 cells, following repeated series of centrifugations at $2000 \mathrm{rpm}, 5 \mathrm{~min}$ each. Then, $24 \mathrm{~h}$ after the pellet formation, MNPs suspended in basic medium were added to the cells, followed by another series of centrifugation to concentrate the MNPs nearby the cell pellet. Culture medium with the MNPs suspension was replaced by fresh basic medium $24 \mathrm{~h}$ post-centrifugations. Fourty-eight (48) hours later, the basic medium was replaced by chondrogenic medium to induce chondrogenic differentiation of hASCs pellets. Chondrogenic medium was composed by Dulbecco's Modified Eagle Medium (DMEM), 1\% A/B, Lproline ( $35 \mathrm{mM}$, Sigma), L-ascorbic acid ( $17 \mathrm{mM}$ ), sodium pyruvate (0.1 M, Alfagene), ITS + 1 (41400-045-insulin-transferrin-selenium liquid media supplement, Sigma), Dexamethasone (1 mM) and human TGF- $\beta 1$ (10 ng/mL, Labclinics).
Chondrogenic cultures were maintained for up to 28 days, and medium changed twice a week. Samples for MTS, DNA and histological assays were collected at 7, 14, 21 and 28 days. Pellets cultured in basic medium were considered a negative control of hASCs chondrogenic differentiation.

MNPs were used to potentially induce osteogenic and chondrogenic differentiation of hASCs through mechanical stimulation provided by the actuation of an external magnetic field (MF).

After the incorporation of MNPs in hASCs, cells were cultured as 2D monolayer in multi-well plates for osteogenic differentiation or as 3D pellets for chondrogenic differentiation. In the case of 3D cell cultures, after the pellets assembly they were carefully collected and transferred into multi-well plates.

Cell monolayers and cell pellets were assessed in static and dynamic conditions so that the influence of the actuation of an external magnetic field could be evaluated in the osteogenic and chondrogenic differentiation processes. Cells incorporating MNPs under static conditions were cultured in the absence of a magnetic stimulus and were defined as a control on the MNPs influence in the process of hASCs differentiation. Under dynamic conditions, the multi-well plates containing cells incorporating MNPs were placed on a magnetic device (Magnefect-nano-II duo ${ }^{\mathbb{R}}$, Nanotherics), which provided the magnetic stimulus. This apparatus operates at $2 \mathrm{~Hz}$ frequency and $0.2 \mathrm{~mm}$ displacement, whose standard values were considered in our experiment $[14,15]$. The mechanical stimulation provided by the oscillation frequency was permanent, being maintained without interruption for 28 days in the chondrogenic study, and 21 days in the osteogenic study.

\subsection{Histological assessment of hASCs differentiation}

Samples collected at each time-point (7, 14, 21 and/or 28 days) were characterized histologically. After a PBS rinse, samples in monolayer culture (osteogenic cultures) and in pellet system were fixed using $10 \%$ formalin solution (Bio-Optica Milano S.p.a) for 30 min after which formalin solution was replaced by PBS. Samples were kept at $4{ }^{\circ} \mathrm{C}$ until further use.

\subsubsection{Alizarin Red staining}

Alizarin Red staining was performed, to assess the extracellular matrix (ECM) mineralization, a later marker for osteogenic differentiation. Alizarin Red solution (ARS, Sigma) was prepared in distilled water $(0.02 \mathrm{~g} / \mathrm{mL})$, and $\mathrm{pH}$ was adjusted to approximately 4.2 , using a $10 \%$ hydroxide solution (Sigma). Cells were incubated with ARS solution for $10 \mathrm{~min}$, and rinsed in PBS to remove unbound staining. Cells were visualized on a Stereo Microscope (Zeiss, Stami 2000-C) and images were collected using a digital camera (Cannon 7).

\subsubsection{Cartilage selective stainings}

After fixation with $10 \%$ formalin, chondrogenic pellets were dehydrated and embedded in paraffin blocks. Sections were cut at $3.5 \mu \mathrm{m}$ with a microtome (Microm HM355S Inopat). Toluidine blue, Safranin-O and Alcian blue were performed to detect components naturally present in cartilage ECM (glycosaminoglycans (GAGs) and proteoglycans (PG)). The presence and or deposition of these molecules relates to the chondrogenic commitment of hASCs previously incorporated with MNPs.

Immediately before incubation with selective stainings, samples were deparaffinized using xylene and hydrated using decreasing alcohol concentration solutions using an automatic stainer equipment (Microm, HMS740).

Toluidine blue was prepared by the dissolution of $1 \%$ of toluidine blue (Carlroth) in distilled water. Pellet sections were incubated with Toluidine blue solution for $2 \mathrm{~min}$, followed by a washing step. 
Safranin-O solution was prepared by dissolving Safranin-O (Sigma) in distilled water $(0.001 \mathrm{~g} / \mathrm{mL})$. A Fast green solution (Sigma) was prepared by dissolving Fast green in distilled water $(0.002 \mathrm{~g} / \mathrm{mL})$. Sections of the pellets were immersed in $0.02 \%$ fast green staining solution for 5 min, following 3 dips in $1 \%$ acetic acid solution. Slides were then immersed in $0.1 \%$ Safranin-O for $5 \mathrm{~h}$.

Alcian blue was prepared by dissolving Alcian blue (Sigma) in acetic acid $(0.3 \mathrm{~g} / \mathrm{mL})$. Pellet sections were immersed in $1 \%$ Alcian blue solution for $1 \mathrm{~h}$, followed by a 5 min washing step.

After toluidine blue, Safranin-O and Alcian blue stainings, slides were dehydrated with a series of alcohol concentration solutions, mounted, and visualized in a transmitted and reflected light microscope (Zeiss, Imager Z1M).

\subsection{Statistical analysis}

All data is represented as mean \pm standard deviation (SD). Statistical analysis was performed using two-tailed Student's $t$-test performed in Microsoft Excel, considering a confidence interval of 95\%. $P$-values lower than 0.05 were considered significant. Both osteogenic and chondrogenic studies were repeated 3 times $(n=3)$, each experiment with a minimum of 3 samples for each assay and time point.

\section{Results}

\subsection{Assessment of MNPs stability in the culture medium}

The MNPs were easily dispersed and colloidally stable in the culture medium over a period of $24 \mathrm{~h}$, as shown by negligible changes in the turbidity measurements (Fig. 1).

\subsection{2 Determination of MNPs internalization in hASCs}

\subsubsection{Microscopy analysis}

After an overnight incubation, MNPs were incorporated to the cells as evidenced by fluorescence microscopy images (Fig. 2A and B). Moreover, the MNPs were homogeneously distributed among the cells and each cell seems to incorporate several MNPs.

The Prussian blue, a typical marker for iron detection, was observed in all stained hASCs, and was preferentially accumulated, as verified by the blue staining, within the cytoplasm of hASCs (Fig. 2C). These data are also supported by SEM and EDS analyses (Fig. 2D and E). SEM micrographs of hASCs incubated with MNPs evidenced that cells incorporated a large amount of these particles that tend to be distributed preferably around the nuclei of hASCs. Moreover, EDS analysis revealed the presence of iron (Fe) at the surface of hASCs after the incubation with MNPs.

\subsubsection{ICP spectrometry}

The incorporation efficiency of MNP within the cells was analyzed and measured by subtracting the initial concentration of MNPs added to the culture medium $(370 \mu \mathrm{g} / \mathrm{mL})$ to the ICP values assessed for iron released to the culture medium after ASCs incubation with the MNPs. The calculated value indicated that a percentage of iron of $63.315 \pm 0.74$ was successfully incorporated into the hASCS.

\subsection{Assessment of cell viability (MTS assay) and cell content (double strand DNA quantification assay)}

\subsubsection{MTS assay}

Cellular viability of hASCs incorporated with MNPs was assessed in osteogenic cultures by MTS assay (Fig. 3).

After 14 days in basic medium culture, static conditions

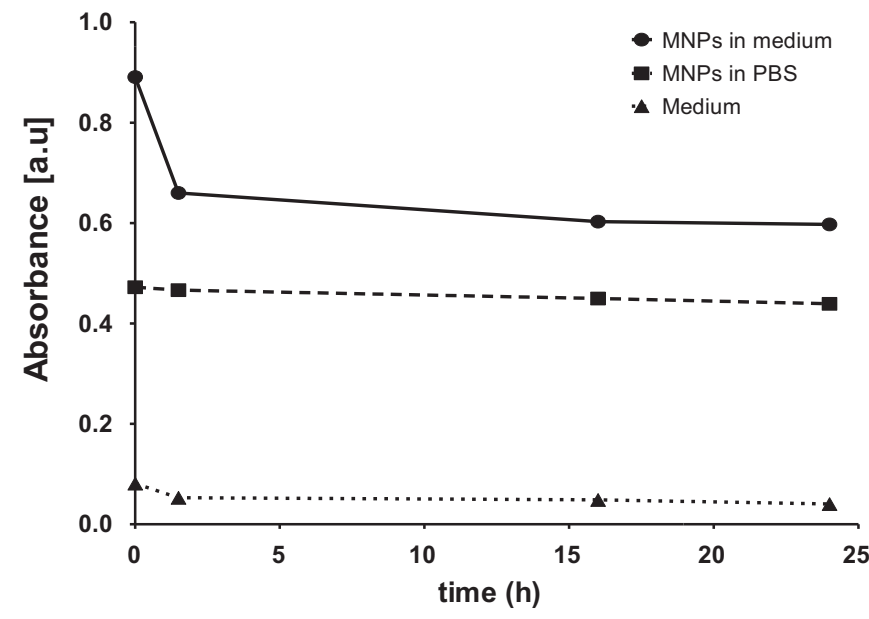

Fig. 1. Spectroscopy at $450 \mathrm{~nm}$ of a MNPs suspension in PBS and in basic culture medium monitored over a period of $24 \mathrm{~h}$, namely $0,1.5,16$ and $24 \mathrm{~h}$. Medium relates to the MNP-free control solution of the assay.

exhibited significant $(P<0.05)$ higher viability levels than those under the magnetic field stimulation generated by the magnetic bioreactor apparatus.

As in basic cultures, in osteogenic supplemented cultures hASCs viability tends to increase with the time in culture. A significant $(P<0.05)$ increase was observed in cell viability of osteogenic cultures in comparison to hASCs cultured in basic medium at 7,14 and 21 days under the influence of an external magnetic stimulus. Nevertheless, the highest metabolic values of all studied conditions were assessed after 21 days in hASCs incorporating MNPs in the absence of magnetic stimulation.

In a similar way, MTS assay was also evaluated in chondrogenic cultures as observed in Fig. 4.

Cell viability values tend to increase in both basic and chondrogenic cultures with the time in culture, with a higher viability increment found in hASCs cultured in basic medium. Independently of the medium used to culture hASCs incorporated with MNPs, the application of a magnetic field (dynamic conditions) did not evidence statistically significant differences $(P>0.05)$ in cell viability.

\subsection{2. dsDNA assay}

Double strand (ds) DNA was assessed and quantified as a mean to determine cell content and proliferation in both osteogenic (Fig. 5) and chondrogenic (Fig. 6) cultures.

The increment in cellular proliferation with the time in culture follows the trend observed for hASCs viability.

Furthermore, osteogenic cultures showed higher cell content and consequently higher proliferation levels in both static and dynamic conditions at day $21(P<0.05)$. In both basic and osteogenic supplemented medium cultures, hASCs proliferation tended to increase during the experimental setup, being statistically significant $(P<0.05)$ from day 7 to day 21 , when a magnetic field stimulus is applied.

Overall, no significant differences were detected in cell content cultured with or without magnetic stimulation by an external magnetic field $(P<0.05)$, despite the fact that the viability assay indicated that hASCs cultured in the absence of an external magnetic stimulus showed higher viability levels after 14 days in basic medium and after 21 days in osteo medium, respectively.

The chondrogenic cultures also correlated with the tendency found in the results of MTS assay. dsDNA quantification showed a slight tendency to increase in time, suggesting that cell proliferation is increasing up to 28 days, independently of the actuation of a magnetic field over the MNPs incorporated in hASCs $(P<0.05)$ 

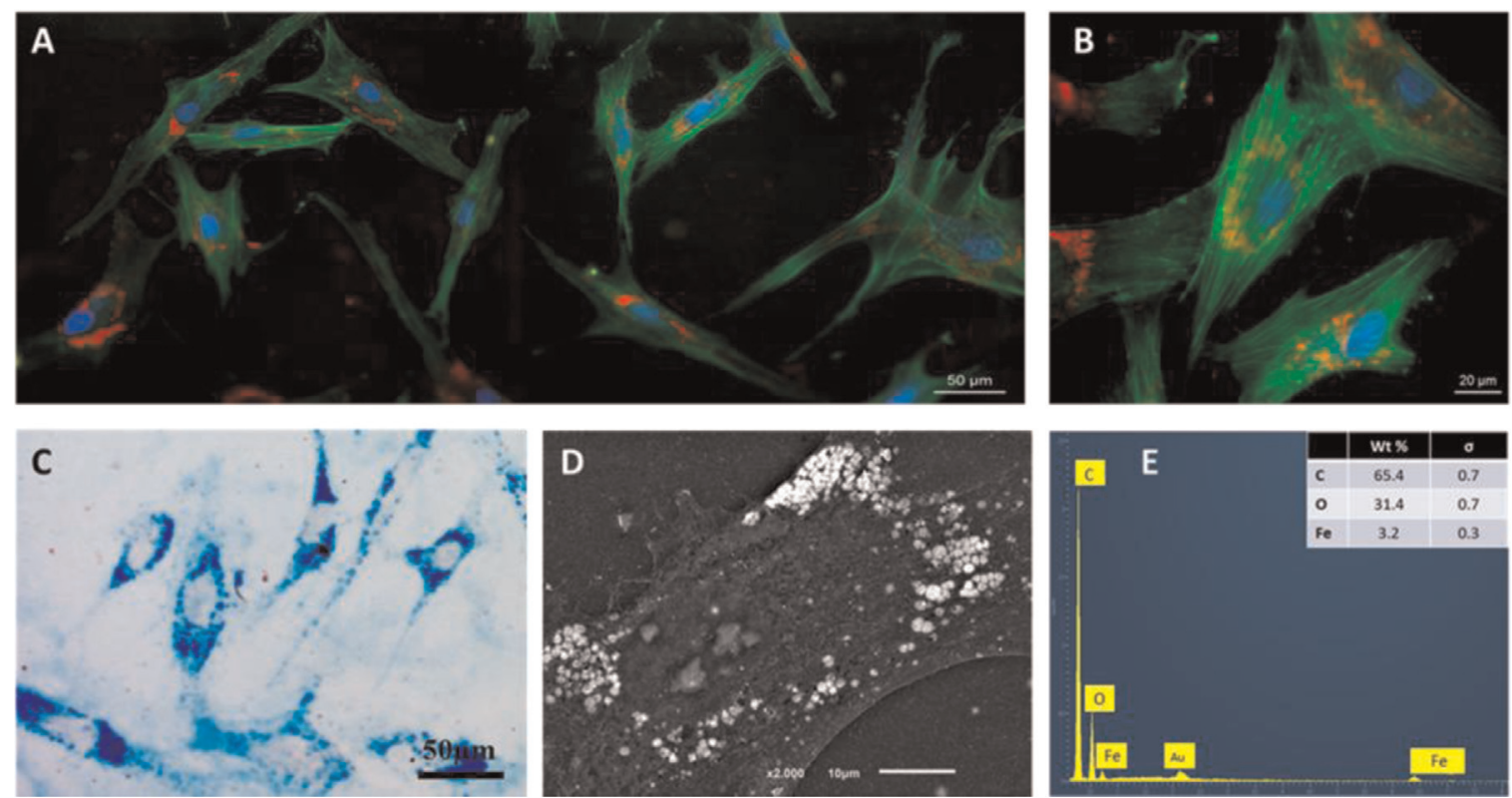

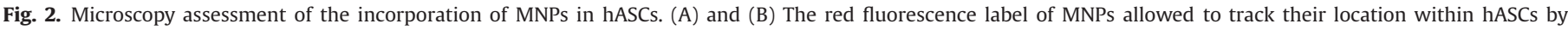

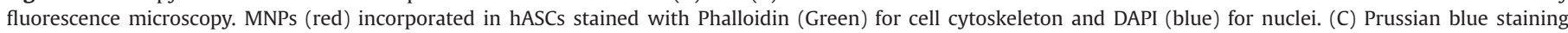

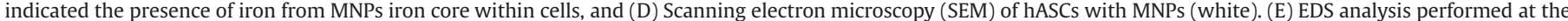

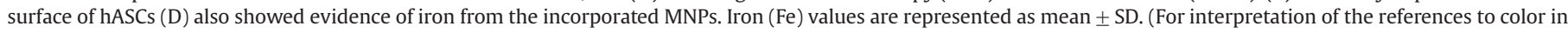
this figure legend, the reader is referred to the web version of this article.)

(Fig. 6).

\subsection{Histological assessment of hASCs differentiation}

\subsubsection{Bone selective staining-Alizarin Red}

Alizarin Red stain was performed on hASCs incorporated with MNPs after 7, 14 and 21 days (Fig. 7).

In control cultures (basic medium), no positive staining was observed in hASCs at the early times of cell culture. However, at day 21 a mild staining (red) is detected under the stimulation of a magnetic field externally generated by the magnetic bioreactor.

In osteogenic cultures, Alizarin Red is not observable until day 14 , either with or without the actuation of an external magnetic field. Yet, a positive staining against calcium deposits was observed in days 14 and 21 in both static and dynamic conditions. Moreover, when a magnetic field was applied (dynamic conditions), the staining was more intense and more widely distributed.

\subsubsection{Cartilage selective stainings}

Toluidine blue, Safranin-O and Alcian blue stains were selected to detect the presence of biomolecules such as glycosaminoglycans (GAGs) and proteoglycans (PGs) naturally synthesized in a cartilaginous-like extracellular matrix (ECM) (Figs. 8, 9 and 10).

When chondrogenic medium was used to supplement hASCs, toluidine blue staining was more intense at day 7 and 21 . The staining is more intense in pellets cultured in chondrogenic medium up to 21 days in the absence of an external magnetic field. By 28 days, the staining is visible in all conditions except in chondrogenic static cultures. When the stimulus of a magnetic field is applied, the stain appears less intense in later time points, mostly at day 21 (Fig. 8).

Pellets were also stained in Safranin-O that showed a more intense staining up to day 21 , when chondrogenic medium was provided to hASCs incorporated with MNPs. Also, under the actuation of an external magnetic field, Safranin-O seems to be more intense at day 21 and 28 than in the absence of the magnetic stimulus (Fig. 9). a

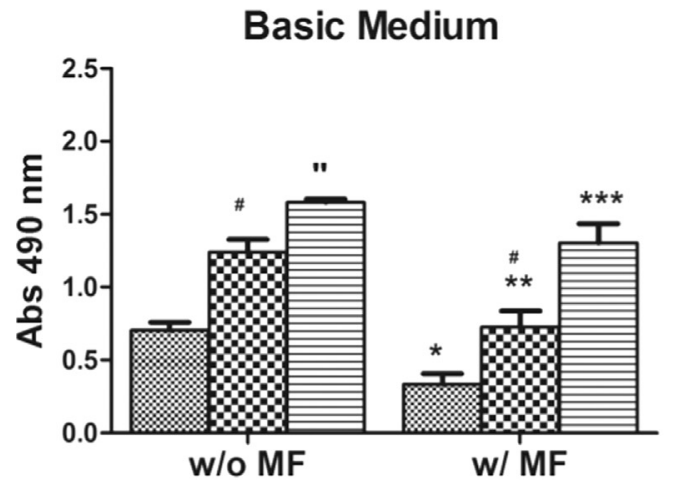

b

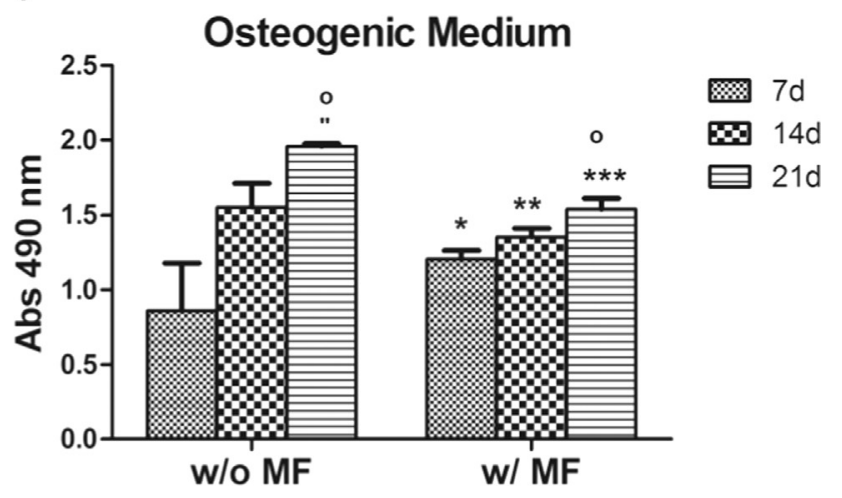

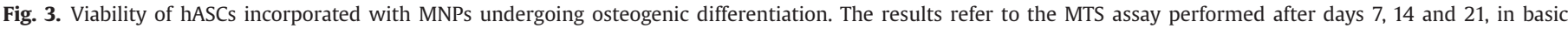

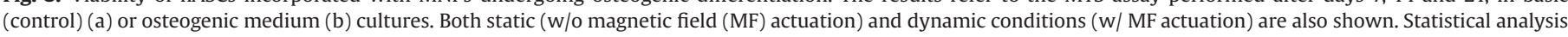

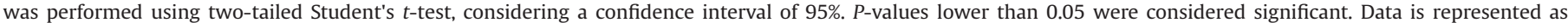
mean \pm SD. All symbols represent statistically significant $(P<0.05)$ differences. 
a

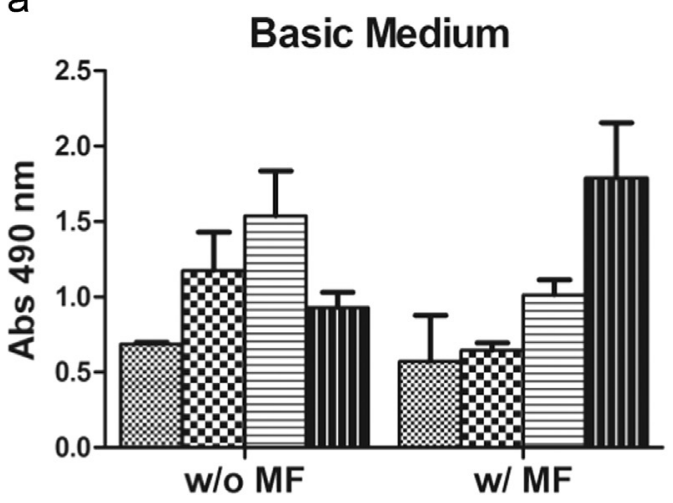

b

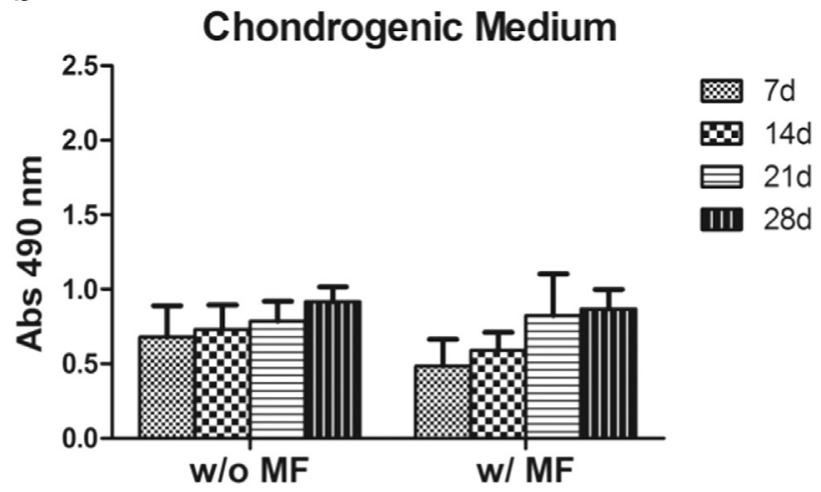

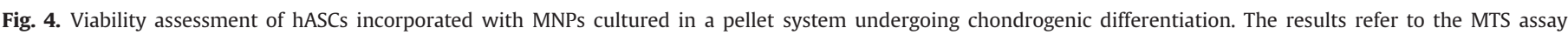

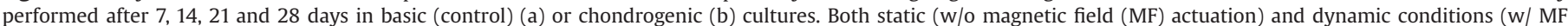

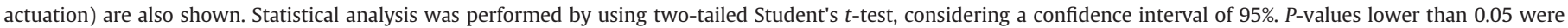
considered statistically significant. Data is represented as mean $\pm \mathrm{SD}$.

Although Alcian blue staining results were not evident in the assayed conditions, GAGs detection is better observed in cultures supplemented with chondrogenic medium. Pellet cultures seem to be less stained under magnetic stimulation, especially in the early days of culture (Fig. 10).

\section{Discussion}

Ongoing research on adult stem cells has predominantly focused on MSCs found within the bone marrow stroma. However, adipose tissue is also derived from the embryonic mesenchyme and growing evidence suggests that adipose tissue is a good source of stem cells [16] with potential for regenerative medicine strategies of musculoskeletal tissues, including bone and cartilage tissues.

This study proposes to investigate the influence of MNPs incorporated in hASCs in the process of chondro- or osteo-genic differentiation with or without the application of an external mechano-magnetic stimulation generated by a magnetic bioreactor. This apparatus stimulates cells with the actuation of a magnetic field, thus providing a magnetic stimulus to be controlled and adjusted to a cellular scale, and to trigger MNPs incorporated in hASCs. The magnetic field is thought to activate intracellular pathways that can convert external mechanical stresses into intracellular biochemical cues and direct cell differentiation. Moreover, a magnetic force-based approach has the leverage of a remote control with spatial and/or temporal precision [17].

Stem cells isolated from human adipose tissue were incorporated with MNPs and differentiated towards osteogenic and chondrogenic lineages, using osteogenic or chondrogenic supplements in culture media under an actuation of a magnetic stimulus provided by an external magnetic field (MF).

Since supplemented culture media are a standardized mean to biochemically induce osteo- and chondro-genic differentiation [18-21], adipose-derived stem cells (hASCs) were also cultured in basic medium, as a control of the differentiation potential of hASCs.

Although the MNPs are commercialized in a PBS suspension, a turbidity assay indicated that MNPs were also stable in culture medium. These particles were easily incorporated within hASCs, as supported by Prussian blue stain and by fluorescence and scanning electron microscopy analysis. Moreover, viability assays suggest that MNPs incorporated by hASCs do not jeopardize the integrity of cellular membranes and these results are supported by the literature [22,23]. Overall, hASCs maintained good viability levels in both basic and supplemented culture media throughout the experimental setup.

Besides MTS assay, DNA quantification was also assessed as a complementary analysis and to provide additional information on the influence of MNPs with or without the actuation of a remote a

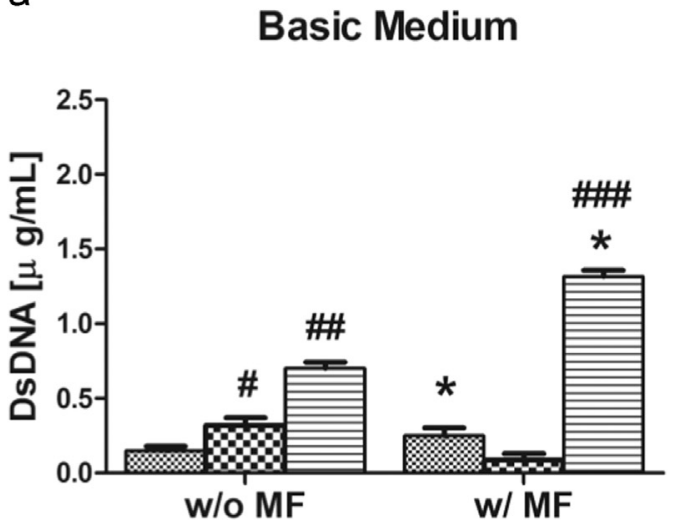

b

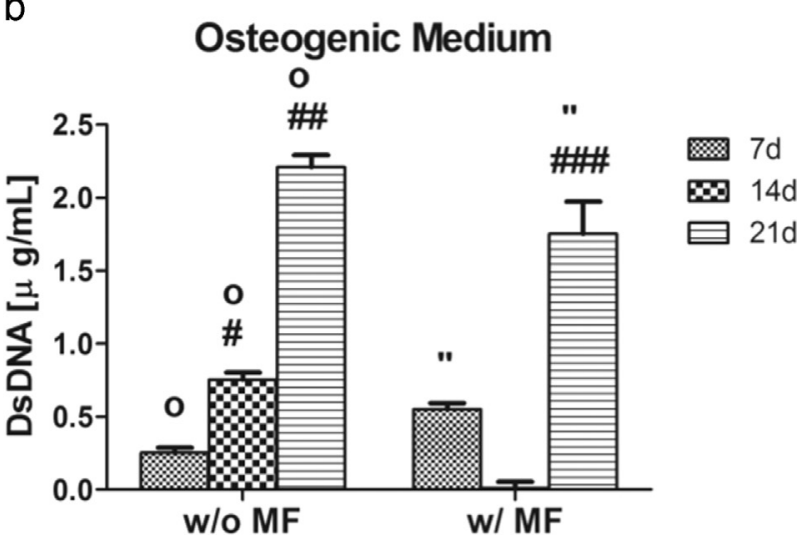

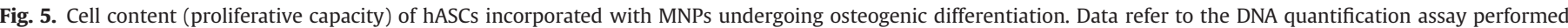

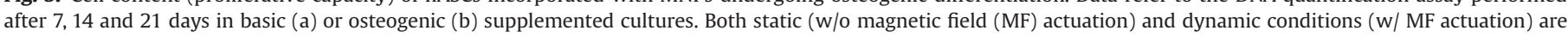

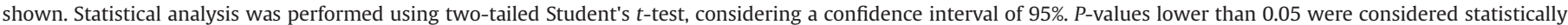
significant. Data is represented as mean \pm SD. All symbols represent significant $(P<0.05)$ differences. 
a

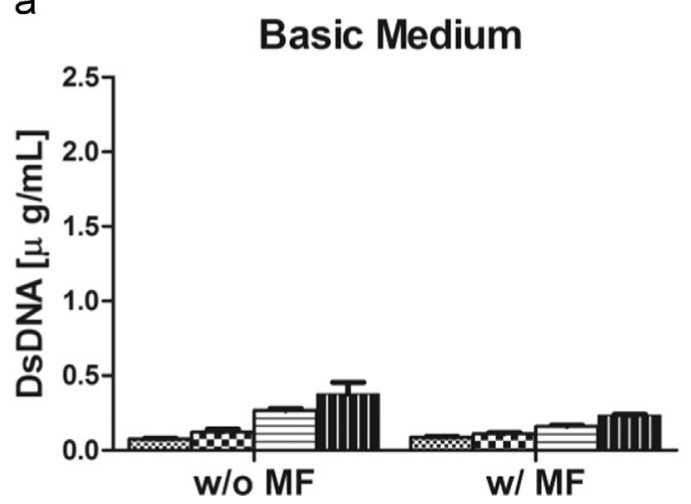

b

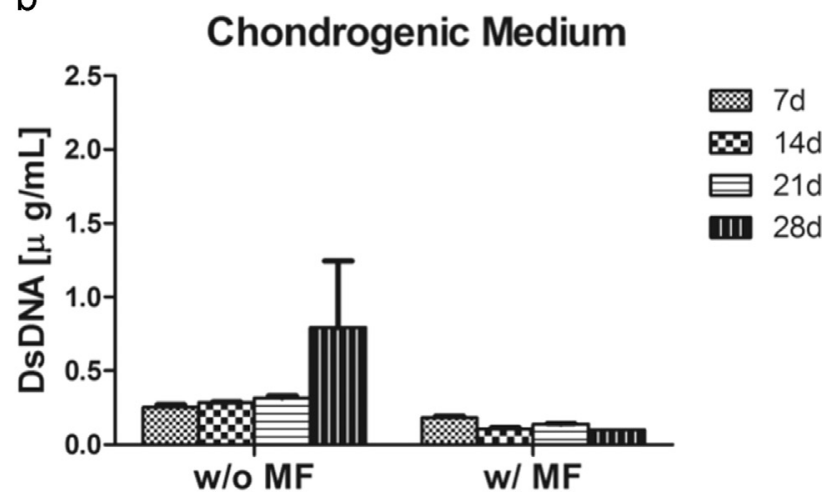

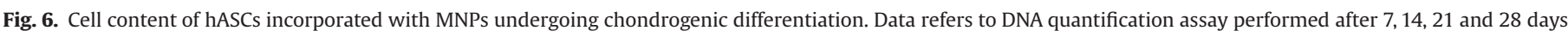

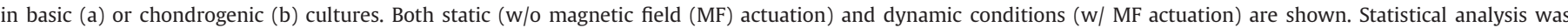

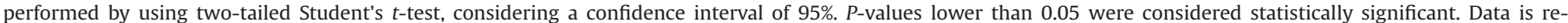
presented as mean \pm SD.

magnetic stimulus towards hASCs differentiation. While MTS assay aimed to assess cell metabolic activity, DNA quantification provided evidence on cell content and consequently on cell proliferation. Although cells need to be viable to duplicate and proliferate, non-proliferative cells can be viable. Cellular differentiation is frequently associated to the maintenance of proliferative values as differentiation implies that a significant part of the cell energy is directed to the production of specific proteins and other biomolecules of the extracellular matrix [24,25].

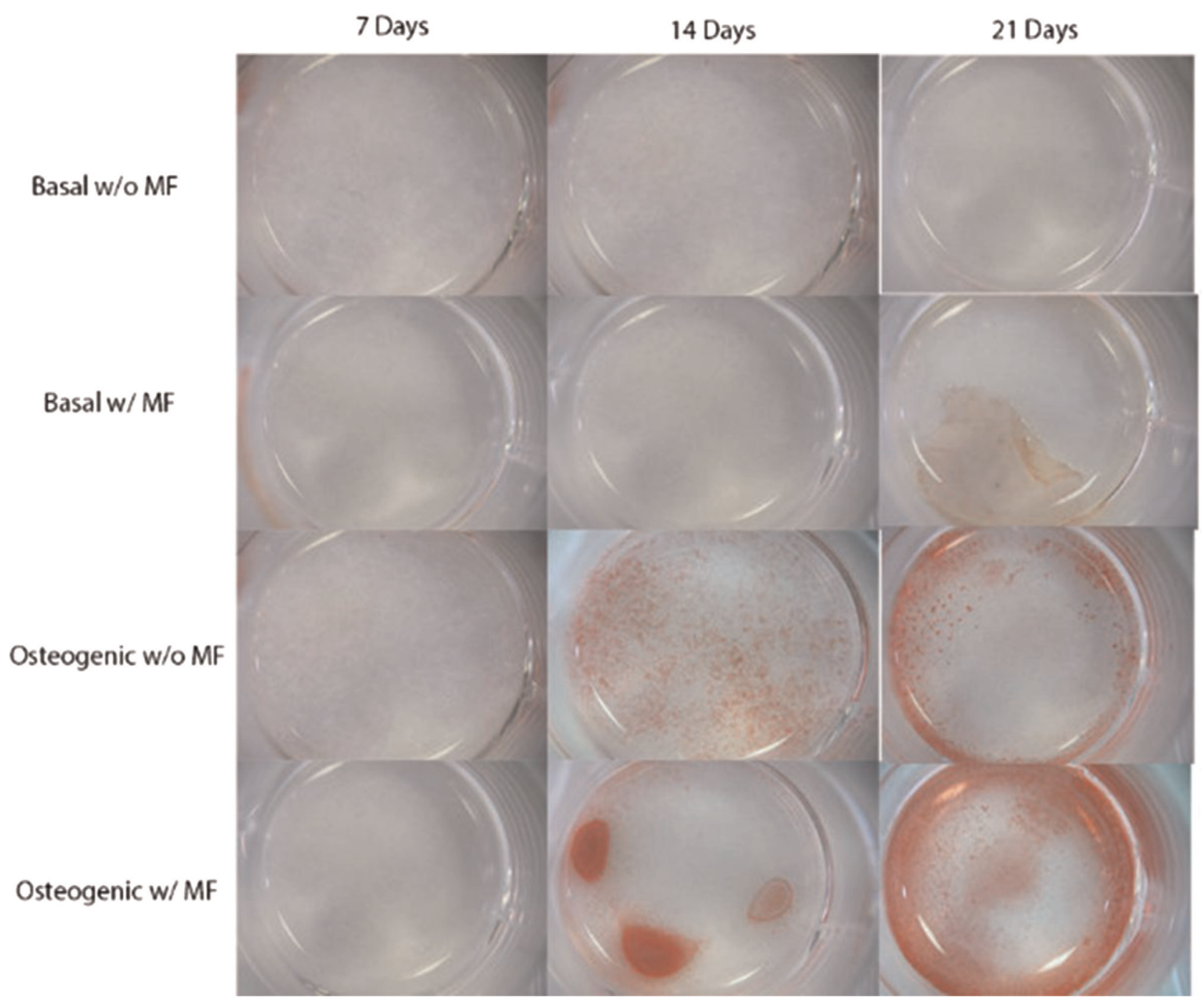

Fig. 7. Alizarin Red staining on hASCs incorporated with MNPs in basic and osteogenic supplemented cultures. Both static (w/o magnetic field (MF) actuation) and dynamic conditions (w/MF actuation) are also shown. Basic medium cultures were defined as experimental controls. 
7 Days

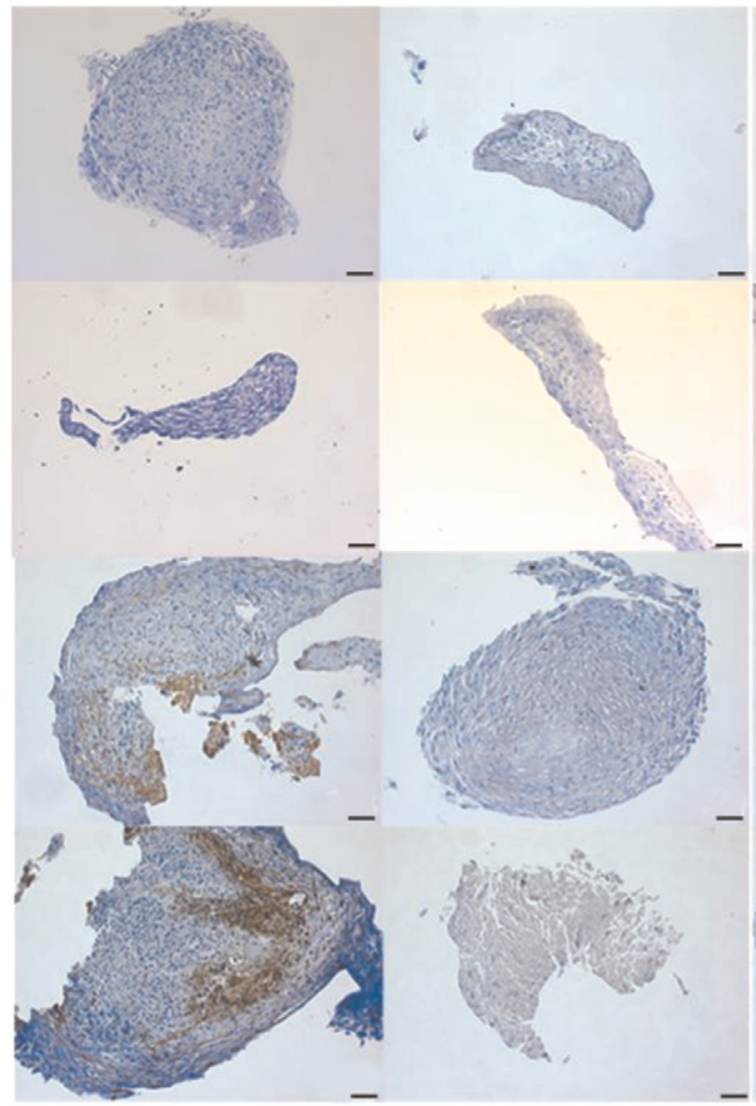

21 Days

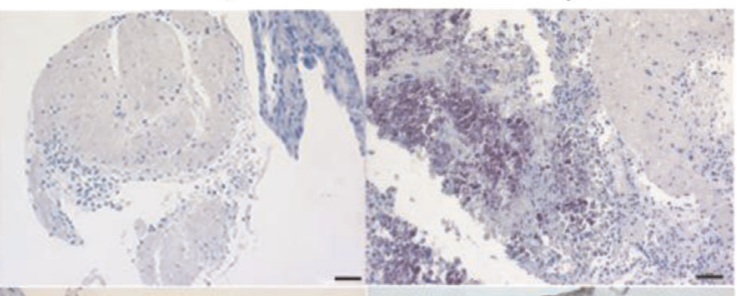

Basal w/o MF

Basal w/ MF

Chondrogenic w/o MF

Chondrogenic W/ MF

. 8. Toluidine blue staining of hASCs incorporated with MNPs cultured in pellet systems. Both static (w/o magnetic field (MF) actuation) and dynamic conditions (w/ MF

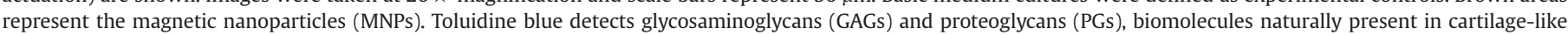
ECM.

\subsection{Osteogenic differentiation of hASCs incorporated with MNPS}

When basic and osteogenic culture conditions are compared, results show that at later times points (day 21), cells exhibit higher levels of viability than those from basic medium, in static conditions, indicating as expected that osteogenic medium also supports cells viability when incorporating MNPs. The increment in cell viability associated to the presence of osteogenic supplements in the culture medium and the exposure to an external magnetic field suggests a synergistic effect between osteogenic supplements and applied magnetic stimulus on hASCs viability towards the osteogenic phenotype.

In terms of cell content, higher values were found in cells cultured in osteogenic medium, especially at later time points. Moreover, these values tend to increase with time independently of the actuation of an external magnetic field. Despite the fact that cells proliferate under the stimulus provided by the magnetic field during the time in culture, osteogenic supplements seem to have a higher influence on cell proliferation when MNPs are incorporated in hASCs that the activation/stimulation of MNPs through the application of an external magnetic field.

Overall, the incorporation of MNPs and the magnetic field actuation do not compromise cellular viability and proliferation levels, two basic requirements for cell based TE approaches.

In this study, we hypothesized that the application of a magnetic field could stimulate and improve hASCs differentiation towards the osteogenic phenotype. Thus, alizarin red staining (ARS) was investigated, as it binds to calcium salts, and is often used to demonstrate the presence of a calcified matrix. Since calcified matrices are characteristic of bone tissues, ARS provides important information concerning the osteogenic potential of hASCs incorporated with MNPs.

As expected, ARS was not observed in basic medium, which is often used for cell expansion, thus not appropriate for osteogenic differentiation. After 14 days in osteogenic cultures, ARS is detectable and tends to increase in intensity and distribution by day 21 . Since the matrix mineralization is a later stage of the osteogenic differentiation process, in early time points (day 7) no matrix mineralization has occurred yet, as literature supports [26]. The staining is more intense and more widely distributed under the stimulus of an external magnetic field. These results showed that the osteogenic supplementation induced the osteogenic differentiation of hASCs as previously reported in the literature [27], but also that magnetic field stimulation participates in the osteogenic process, more specifically in terms of the production of a calcium rich ECM. It is also demonstrated that the stimulus provided by the application of an external magnetic field can regulate cell differentiation of hASCs incorporated with MNPs towards the osteogenic lineage. Moreover, these results are in agreement with Kanczler et al. work [28], in which labeled bone marrow mesenchymal stem cells (BM-MSCs) with magnetic beads encapsulated into alginate chitosan capsules were exposed to magnetic forces. The provided mechanical stimulation enhanced proteoglycan, collagen and ECM synthesis and elevated the expression of type-I and type-II collagen [28]. In another study, a significantly higher mineral matrix ratio was detected in BM-MSCs after 3 weeks of magneto-mechanical stimulation combined with osteogenic medium culture [29]. In basic medium cultures, no 


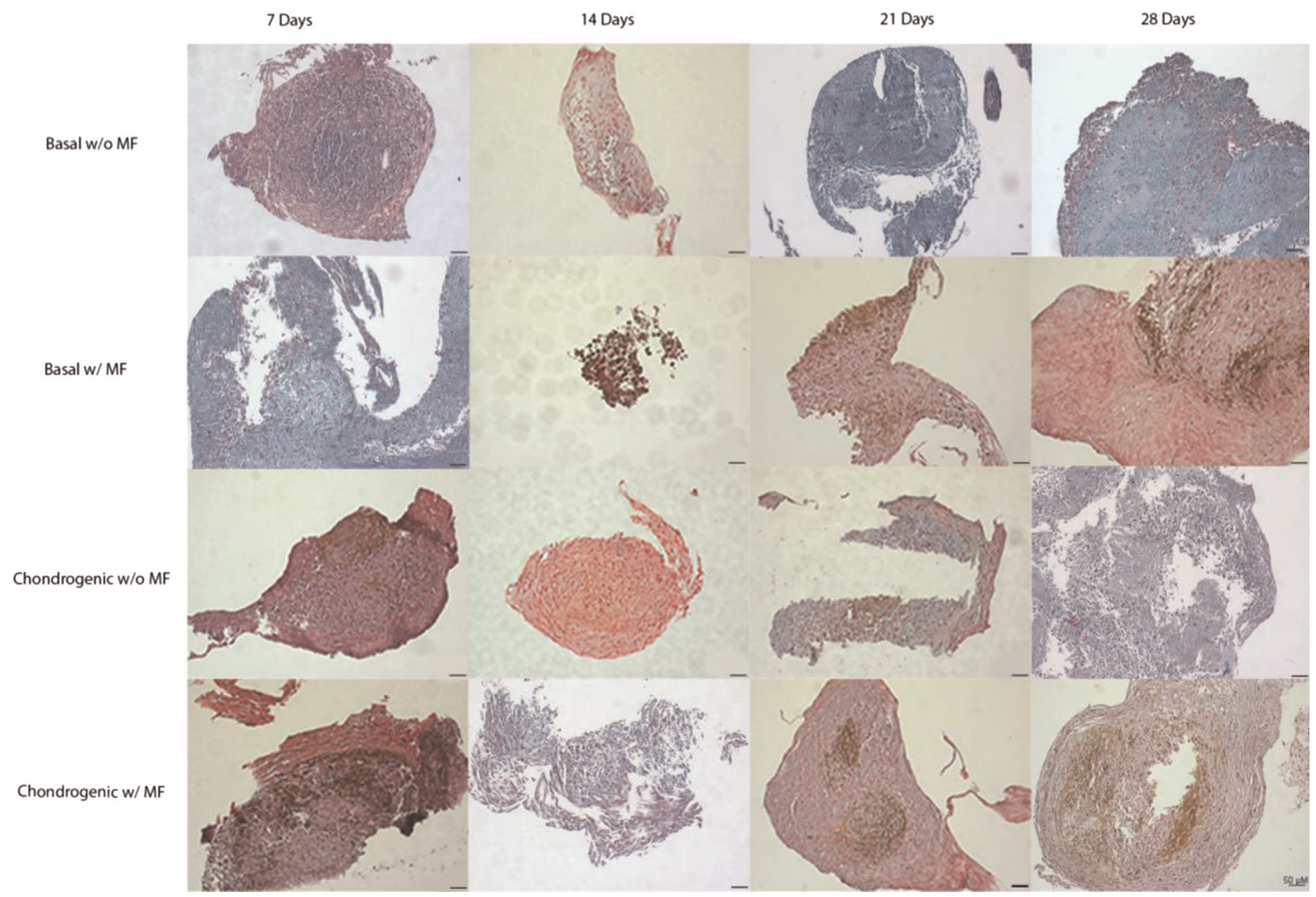

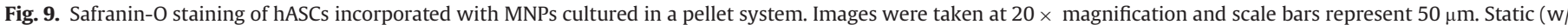

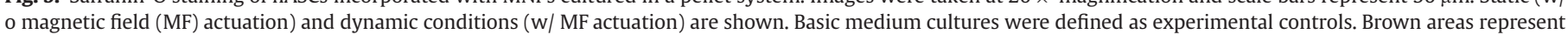
the magnetic nanoparticles (MNPs). Safranin-O detects GAGs and PGs, indicating the presence of cartilage-like ECM.

mineralization occurred [29].

Despite differences between cell sources, and the intrinsic characteristics of the magnetic field (e.g. intensity, frequency and duration) applied for cell stimulation, magnetic forces seem to influence cell behavior, with a particular response in the enhancement of the production of bone-like ECM biomolecules.

\subsection{Chondrogenic differentiation of hASCs incorporated with MNPS}

Viability and proliferation levels of hASCs cultured in pellets in both chondrogenic and basic media are maintained, showing no significant differences up to 28 days in culture. Also, the application of a magnetic field did not particularly influence the chondrogenic pellet cultures. Furthermore, magnetic nanoparticles showed no cytotoxicity effect nor a stimulatory effect towards hASCs incorporating MNPs in pellet systems. The maintenance of the proliferation may be associated with the differentiation mechanism, in which cells undergoing differentiation direct most of their energy and nutrients to this process, compromising the proliferation rate of the cells. The non-cytotoxic effect of MNPs observed in our study is also supported by several studies from the literature [30,31]. Saha et al. labeled human BM-MSCs with iron oxide nanoparticles and viability/proliferation was unaffected for up to 14 days in culture [31].

To determine the influence of MNPs under the application of an external magnetic stimulus in the chondrogenic potential of hASCs, selective stains associated to the detection of GAGs and PGs, typically found in native cartilage, were assessed. Although
Alcian blue results were not conclusive in this study, Toluidine blue and Safranin O stains indicated that chondrogenic medium seems to be more important for the detection of PGs than MNPs incorporated in hASCs or the stimulus provided by the magnetic field actuation. Nevertheless, looking into the Safranin-O results, chondrogenic medium does influence the detection of proteins present in a chondrogenic-like ECM but also does the exposure to a magnetic field in a smaller extent, especially at very early (day 7) and later time points (day 21 and day 28). An enhancement of the chondrogenic differentiation using a magnetic field has also been reported [32]. Amin et al. demonstrated that magnetic force applied into BM-MSCs have a synergistic effect with biochemical factors enhancing chondrogenic differentiation [32]. Also, ChungHwan Chen et al. studied the effect of an electromagnetic field onto chondrogenic differentiation of ASCs, concluding that although viability was not affected, differentiation was enhanced when the electromagnetic field was applied to the cells [33].

\section{Conclusions}

Musculoskeletal impairments have a great impact in public health and current clinical procedures fail to reach an ideal healing solution with complete restoration of function. Magnetic nanoparticles (MNPs) based strategies are promising candidates to treat musculoskeletal tissues, particularly bone and cartilage tissues, through stimulation at the cellular level, which could influence intracellular pathways and impact cell behavior, including stem 


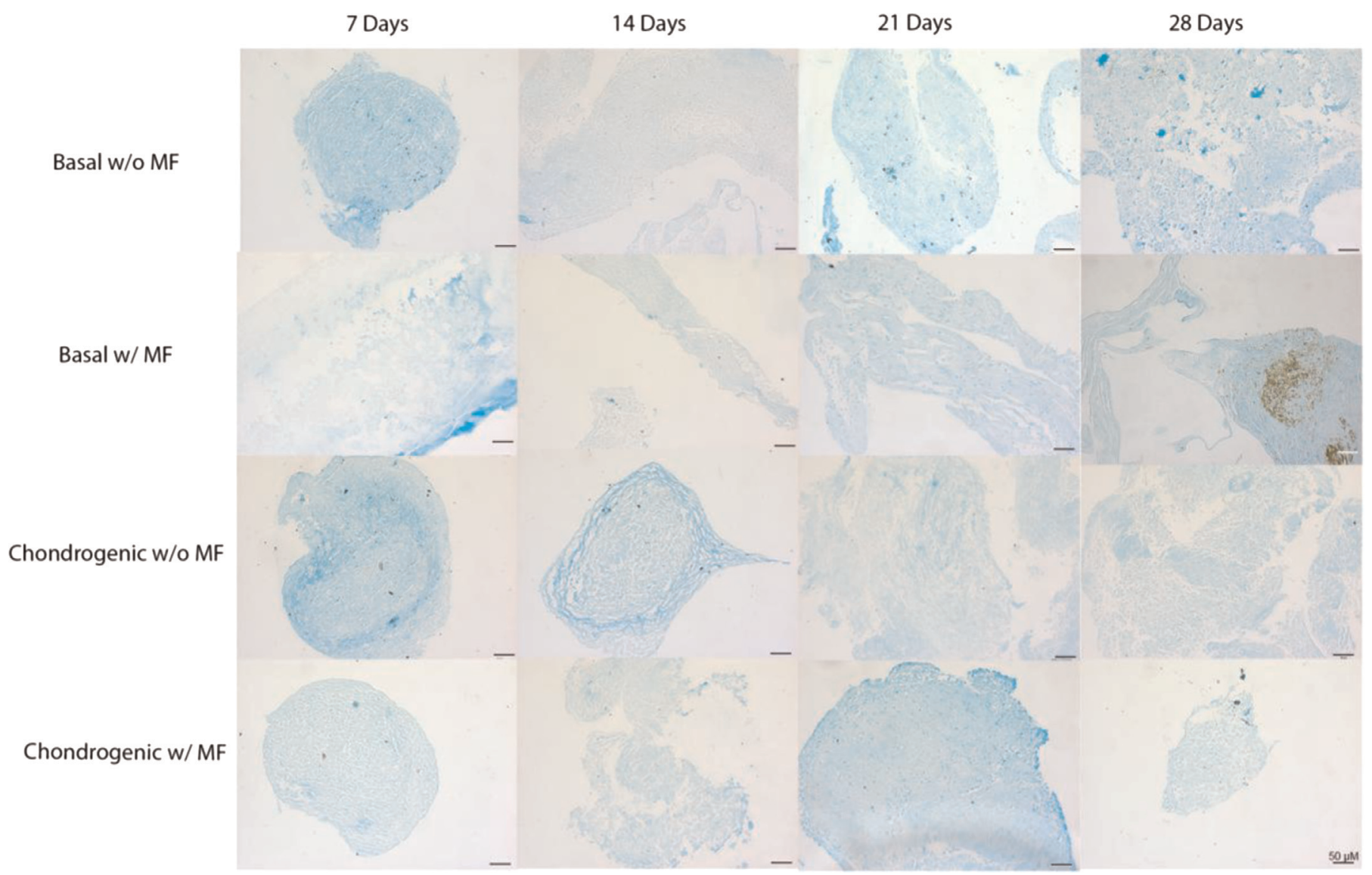

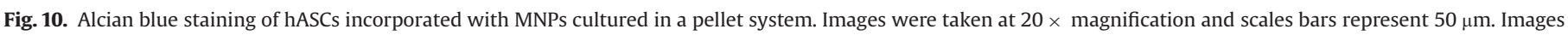

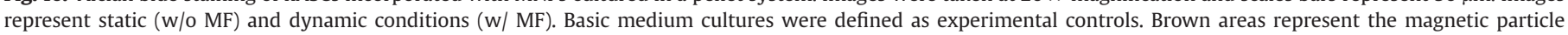
aggregates accumulated within the pellet. Alcian blue highlights the presence of GAGs (blue), typically found in cartilage-like ECM.

\section{cell differentiation process.}

Using nanoparticles with an iron oxide core in combination with the application of an external magnetic stimulus, the differentiation of adipose derived stem cells (hASCs) was assessed through lineage specific stains. Although complementary studies are necessary to better understand the mechanism of action of MNPs in the process of hASCs differentiation, the results obtained in this work indicate that MNPs under the influence of a magnetic field have a greater impact in the osteogenic differentiation of hASCs than in stimulating hASCs into the chondrogenic phenotype. Moreover, this work also suggests that a system involving the use of MNPs and the application of an external magnetic field stimulation is a promising candidate for bone and cartilage differentiation strategies with envisioned potential for alternative treatment approaches aiming at the regeneration of bone and cartilage defects.

\section{Acknowledgments}

This research was supported by Project PEst-C/SAU/LA0026/ 2013/; NORTE-01-0124-FEDER-000020 under ON.2SR\&TD Integrated Program; 7th Framework Programme (FP7/2007-2013) under grant agreement no. REGPOT-CT2012-316331-POLARIS.

\section{References}

[1] M.M. Stevens, Biomaterials for bone tissue engineering, Materialstoday 11
(2008) 18-25.

[2] J.M. Holzwarth, P.X. Ma, Biomimetic nanofibrous scaffolds for bone tissue engineering, Biomaterials 32 (2011) 9622-9629.

[3] E.H. Lim, J.P. Sardinha, S. Myers, Nanotechnology biomimetic cartilage regenerative scaffolds, Arch. Plast. Surg. 41 (2014) 231-240.

[4] O. Veiseh, J.W. Gunn, M. Zhang, Design and fabrication of magnetic nanoparticles for targeted drug delivery and imaging, Adv. Drug Deliv. Rev. 62 (2010) 284-304.

[5] A. Gloria TR, U. D’Amora, S. Zeppetelli, T. D’Alessandro, M. Sandri, M. BañobreLópez, Y. Piñeiro-Redondo, M. Uhlarz, A. Tampieri, J. Rivas, T. Herrmannsdörfer, V.A. Dediu, L. Ambrosio, R. De Santis, Magnetic poly( $\varepsilon$-caprolactone)/irondoped hydroxyapatite nanocomposite substrates for advanced bone tissue engineering, J. R. Soc. 10 (2014) 20120833.

[6] R. Ogawa, H. Mizuno, A. Watanabe, M. Migita, T. Shimada, et al., Osteogenic and chondrogenic differentiation by adipose-derived stem cells harvested from GFP transgenic mice, Biochem. Biophys. Res. Commun. 313 (2004) 871-877.

[7] P.A. Zuk, M. Zhu, P. Ashjian, D.A. De Ugarte, J.I. Huang, et al., Human adipose tissue is a source of multipotent stem cells, Mol. Biol. Cell 13 (2002) 4279-4295.

[8] J.I. Huang, N. Kazmi, M.M. Durbhakula, T.M. Hering, J.U. Yoo, et al., Chondrogenic potential of progenitor cells derived from human bone marrow and adipose tissue: a patient-matched comparison, J. Orthop. Res. 23 (2005) 1383-1389.

[9] T. Mochizuki, T. Muneta, Y. Sakaguchi, A. Nimura, A. Yokoyama, et al., Higher chondrogenic potential of fibrous synovium- and adipose synovium-derived cells compared with subcutaneous fat-derived cells: distinguishing properties of mesenchymal stem cells in humans, Arthritis Rheum. 54 (2006) 843-853.

[10] T. Rada, R.L. Reis, M.E. Gomes, Distinct stem cells subpopulations isolated from human adipose tissue exhibit different chondrogenic and osteogenic differentiation potential, Stem Cell Rev. 7 (2011) 64-76.

[11] A. Petri-Fink, B. Steitz, A. Finka, J. Salaklang, H. Hofmann, Effect of cell media on polymer coated superparamagnetic iron oxide nanoparticles (SPIONs): colloidal stability, cytotoxicity, and cellular uptake studies, Eur. J. Pharm. Biopharm. 68 (2008) 129-137.

[12] X. Yang, E. Bakaic, T. Hoare, E.D. Cranston, Injectable polysaccharide hydrogels reinforced with cellulose nanocrystals: morphology, rheology, degradation, and cytotoxicity, Biomacromolecules 14 (2013) 4447-4455.

[13] E.G Popa, S.G. Caridade, J.F. Mano, R.L. Reis, M.E. Gomes, Chondrogenic 
potential of injectable kappa-carrageenan hydrogel with encapsulated adipose stem cells for cartilage tissue-engineering applications, J. Tissue Eng. Regen. Med. 9 (2013) 550-563.

[14] A. Fouriki, M.A. Clements, N. Farrow, J. Dobson, Efficient transfection of MG-63 osteoblasts using magnetic nanoparticles and oscillating magnetic fields, J. Tissue Eng. Regen. Med. 8 (2014) 169-175.

[15] A. Fouriki, N. Farrow, M.A. Clements, J. Dobson, Evaluation of the magnetic field requirements for nanomagnetic gene transfection, Nano Rev. 1 (2010).

[16] A. Patricia, P.A. Zuk Min Zhu, De. Ugarte Daniel A., Huang Jerry I., Mizuno Hiroshi, Alfonso Zeni C., Fraser John K., Benhaim Prosper, Hedrick Marc H., Human adipose tissue is a source of multipotent stem cells, Mol. Biol. Cell 13 (2002) 4279-4295.

[17] E.A. Lee, H. Yim, J. Heo, H. Kim, G. Jung, et al., Application of magnetic nanoparticle for controlled tissue assembly and tissue engineering, Arch. Pharm. Res. 37 (2014) 120-128.

[18] T.M. Liu, M. Martina, D.W. Hutmacher, J.H. Hui, E.H. Lee, et al., Identification of common pathways mediating differentiation of bone marrow- and adipose tissue-derived human mesenchymal stem cells into three mesenchymal lineages, Stem Cells 25 (2007) 750-760.

[19] R.H. Lee, B. Kim, I. Choi, H. Kim, H.S. Choi, et al., Characterization and expression analysis of mesenchymal stem cells from human bone marrow and adipose tissue, Cell. Physiol. Biochem. 14 (2004) 311-324.

[20] J.B. Mitchell, K. McIntosh, S. Zvonic, S. Garrett, Z.E. Floyd, et al., Immunophenotype of human adipose-derived cells: temporal changes in stromal-associated and stem cell-associated markers, Stem Cells 24 (2006) 376-385.

[21] H. Tapp, E.N. Hanley Jr., J.C. Patt, H.E. Gruber, Adipose-derived stem cells: characterization and current application in orthopaedic tissue repair, Exp. Biol. Med. 234 (2009) 1-9.

[22] M.R. Dzamukova, E.A. Naumenko, E.A. Rozhina, A.A. Trifonov, R.F. Fakhrullin, Cell surface engineering with polyelectrolyte-stabilised magnetic nanoparticles: a facile approach for fabrication of artificial multicellular tissuemimicking clusters, Nano Res. (2015) 1-18.

[23] E.A. Naumenko, M.R. Dzamukova, G.I. Fakhrullina, F.S. Akhatova, R.
F. Fakhrullin, Nano-labelled cells-a functional tool in biomedical applications Curr. Opin. Pharmacol. 18 (2014) 84-90.

[24] M.B. Goldring, K. Tsuchimochi, K. Ijiri, The control of chondrogenesis, J. Cell. Biochem. 97 (2006) 33-44.

[25] J.B. Lian, G.S. Stein, Concepts of osteoblast growth and differentiation: basis for modulation of bone cell development and tissue formation, Crit. Rev. Oral Biol. Med. 3 (1992) 269-305.

[26] E. Donzelli, A. Salvade, P. Mimo, M. Vigano, M. Morrone, et al., Mesenchyma stem cells cultured on a collagen scaffold: in vitro osteogenic differentiation, Arch. Oral Biol. 52 (2007) 64-73.

[27] E. Birmingham, G.L. Niebur, P.E. McHugh, G. Shaw, F.P. Barry, et al., Osteogenic differentiation of mesenchymal stem cells is regulated by osteocyte and osteoblast cells in a simplified bone niche, Eur. Cell Mater. 23 (2012) 13-27.

[28] J.M. Kanczler, H.S. Sura, J. Magnay, D. Green, R.O. Oreffo, et al., Controlled differentiation of human bone marrow stromal cells using magnetic nanoparticle technology, Tissue Eng. Part A 16 (2010) 3241-3250.

[29] B. Hu, A.J. El Haj, J. Dobson, Receptor-targeted, magneto-mechanical stimulation of osteogenic differentiation of human bone marrow-derived mesenchymal stem cells, Int. J. Mol. Sci. 14 (2013) 19276-19293.

[30] A. Ito, E. Hibino, H. Honda, K.-i Hata, H. Kagami, et al. A new methodology of mesenchymal stem cell expansion using magnetic nanoparticles, Biochem. Eng. J. 20 (2004) 119-125.

[31] S. Saha, X.B. Yang, S. Tanner, S. Curran, D. Wood, et al., The effects of iron oxide incorporation on the chondrogenic potential of three human cell types, J. Tissue Eng. Regen. Med. 7 (2013) 461-469.

[32] H.D. Amin, M.A. Brady, J.P. St-Pierre, M.M. Stevens, D.R. Overby, et al., Stimulation of chondrogenic differentiation of adult human bone marrow-derived stromal cells by a moderate-strength static magnetic field, Tissue Eng. Part A 20 (2014) 1612-1620.

[33] C.H. Chen, Y.S. Lin, Y.C. Fu, C.K. Wang, S.C. Wu, et al., Electromagnetic fields enhance chondrogenesis of human adipose-derived stem cells in a chondrogenic microenvironment in vitro, J. Appl. Physiol. 114 (2013) 647-655. 